\title{
Aircraft Dynamic Modeling in Turbulence
}

\author{
Eugene A. Morelli ${ }^{1}$ \\ NASA Langley Research Center, Hampton, Virginia, 23681 \\ Kevin Cunningham ${ }^{2}$ \\ NASA Langley Research Center, Hampton, Virginia, 23681
}

\begin{abstract}
A method for accurately identifying aircraft dynamic models in turbulence was developed and demonstrated. The method uses orthogonal optimized multisine excitation inputs and an analytic method for enhancing signal-to-noise ratio for dynamic modeling in turbulence. A turbulence metric was developed to accurately characterize the turbulence level using flight measurements. The modeling technique was demonstrated in simulation, then applied to a subscale twin-engine jet transport aircraft in flight. Comparisons of modeling results obtained in turbulent air to results obtained in smooth air were used to demonstrate the effectiveness of the approach.
\end{abstract}

\begin{tabular}{|c|c|c|}
\hline & & Nomenclature \\
\hline AirSTAR & $=$ & Airborne Subscale Transport Aircraft Research \\
\hline$a_{x}, a_{y}, a_{z}$ & $=$ & body-axis translational accelerometer measurements, $g$ \\
\hline$b$ & $=$ & wing span, $\mathrm{ft}$ \\
\hline$C_{X}, C_{Y}, C_{Z}$ & $=$ & body-axis nondimensional aerodynamic force coefficients \\
\hline$C_{l}, C_{m}, C_{n}$ & $=$ & body-axis nondimensional aerodynamic moment coefficient \\
\hline $\bar{c}$ & $=$ & wing mean aerodynamic chord, $\mathrm{ft}$ \\
\hline GPS & $=$ & global positioning system \\
\hline INS & $=$ & inertial navigation system \\
\hline$I_{x}, I_{y}, I_{z}, I_{x z}$ & $=$ & mass moments of inertia, slug- $\mathrm{ft}^{2}$ \\
\hline$M_{T}$ & $=$ & body-axis pitching moment from engine thrust, $\mathrm{ft}-\mathrm{lbf}$ \\
\hline$m$ & $=$ & aircraft mass, slug \\
\hline$p, q, r$ & $=$ & body-axis roll, pitch, and yaw rates, $\mathrm{rad} / \mathrm{s}$ or $\mathrm{deg} / \mathrm{s}$ \\
\hline $\bar{q}$ & $=$ & dynamic pressure, $\mathrm{lbf} / \mathrm{ft}^{2}$ \\
\hline$S$ & $=$ & wing reference area, $\mathrm{ft}^{2}$ \\
\hline SIDPAC & $=$ & System IDentification Programs for AirCraft \\
\hline$T$ & $=$ & maneuver length, $\mathrm{s}$ \\
\hline$T_{x}, T_{z}$ & $=$ & body-axis components of engine thrust, lbf \\
\hline$V$ & $=$ & true airspeed, $\mathrm{ft} / \mathrm{s}$ \\
\hline$\alpha$ & $=$ & angle of attack, rad or deg \\
\hline$\beta$ & $=$ & sideslip angle, rad or deg \\
\hline$\delta_{e}, \delta_{a}, \delta_{r}$ & $=$ & elevator, aileron, and rudder deflections, rad or deg \\
\hline$\phi, \theta, \psi$ & $=$ & Euler roll, pitch, and yaw angles, rad or deg \\
\hline$\Sigma$ & $=$ & covariance matrix \\
\hline$\|x\|$ & $=$ & Euclidean norm of $x$, or magnitude of complex number $x$ \\
\hline
\end{tabular}

\footnotetext{
${ }^{1}$ Research Engineer, Dynamic Systems and Control Branch, MS 308, Associate Fellow

${ }^{2}$ Research Engineer, Flight Dynamics Branch, MS 308, AIAA Member
} 


$\begin{array}{lll}T & =\text { transpose } \\ -1 & =\text { matrix inverse } \\ \sim & =\text { estimate } \\ \sim & =\text { Fourier transform } \\ & =\text { time derivative } \\ \text { subscripts } & \\ \text { cg } & =\text { center of gravity } \\ o & =\text { reference value or base term }\end{array}$

\section{Introduction}

$\mathrm{T}$ HE goal of stability and control flight testing is to accurately characterize the relationships among aircraft controls and aircraft dynamic responses by identifying mathematical models from measured flight data. When conducting flight test maneuvers to collect data for aircraft dynamic modeling, an unmeasured excitation such as turbulence will give rise to inaccuracy and uncertainty in aircraft dynamic models identified from the measured data. Because turbulence does not scale (turbulence of a given level has greater effect on aircraft response as the scale of the aircraft gets smaller), the adverse effects of turbulence on dynamic modeling results are worse for subscale aircraft than for full-scale aircraft.

One common approach to solving the problem is to conduct stability and control flight testing only in smooth air. Another approach has been to estimate an analytical characterization of the turbulence as a stochastic input or process noise, while simultaneously estimating dynamic model parameters in an output-error formulation. This approach is called the filter-error method ${ }^{1-3}$. The filter-error method requires the use of a Kalman filter to estimate the aircraft states, which are stochastic quantities due to turbulence being included as process noise in the dynamic model. The combination of the Kalman filter, the nonlinear optimization necessary for output-error parameter estimation, the difficulty in analytically characterizing the turbulence, and the inherent nonlinearity of the problem make the filter-error method very difficult to use in practice.

In this paper, a different approach is studied. Instead of attempting to characterize the turbulence analytically and battling the associated complications of incorporating turbulence into the dynamic model, the turbulence is instead dominated or marginalized, so that standard parameter estimation methods can be employed to produce accurate dynamic modeling results. The equation-error method in the frequency domain is particularly well-suited to this task, and can be implemented as a real-time method ${ }^{1}$. Separate work ${ }^{4}$ has studied the possible use of turbulence sensors to convert the turbulence from an unknown excitation input to a known excitation input, after which standard parameter estimation methods could be applied. In that case, the turbulence is being incorporated into the model, but using a measurement rather than an analytical estimation technique. In this work, experimental and analytical methods are applied to significantly reduce the impact of the turbulence, allowing accurate dynamic modeling results to be obtained using standard parameter estimation methods.

The methods were applied to the NASA Langley T-2 subscale jet transport aircraft, described in detail in Section III, and flight tests were carried out using the NASA Langley AirSTAR flight test capability. This aircraft configuration has been tested extensively in the wind tunnel ${ }^{5,6}$, although the wind-tunnel test articles differed in some geometric details from the aircraft used for the flight tests. The wind tunnel data provided an aerodynamic database for a nonlinear aircraft simulation. A linear dynamic model extracted from the nonlinear aircraft simulation was used to validate the effectiveness of the methods developed in this work. The methods were also applied to T-2 flight data in both smooth air and turbulent air at similar flight conditions, to further investigate and validate the effectiveness of the approach.

A technique for evaluating the turbulence level using in-flight measurements was developed to correlate the turbulence level with dynamic modeling results, and also to help specify the control surface excitation amplitudes necessary to reduce the effects of turbulence on the dynamic modeling results. Section IV provides details of the turbulence metric calculation.

The next section describes a method that has been used successfully in the past to design excitation inputs for stability and control flight testing. Then the test aircraft and instrumentation are described, followed by explanation and demonstration of a method for accurately characterizing flight turbulence levels using measured aircraft response data. Next, experimental and analytical methods developed for aircraft dynamic modeling in turbulence 
are described and demonstrated. The methods are applied to the T-2 subscale aircraft, first using simulation data, then using flight data. Finally, the results are discussed and conclusions are presented.

All of the experiment design, data analysis, and modeling tasks included in this work were done using system identification software written in MATLAB ${ }^{\circledR}$, called System IDentification Programs for AirCraft, or SIDPAC ${ }^{1}$. SIDPAC is bundled with Ref. [1], and is therefore publicly available.

\section{Orthogonal Optimized Multisine Input Design}

Stability and control characteristics of a flight vehicle can be quantified by parameters appearing in the equations of motion, such as stability and control derivatives in the case of a linear dynamic model ${ }^{1}$. Values of these parameters can be found from flight data using various forms of least-squares fitting of the model output to measured data ${ }^{1}$, where the model output depends on the model parameters to be estimated. For aerospace vehicles, flight test maneuvers are often executed by applying designed excitation inputs to the control surfaces, using an onboard flight computer ${ }^{1,7-10}$. These inputs are perturbations about the nominal or trim control surface deflections.

Multi-axis orthogonal optimized multisine inputs are effective excitation time series for stability and control flight testing. This section describes how these inputs are designed and why this particular input form is efficient for identifying dynamic models from flight data. More details on this input design technique and flight applications can be found in Refs. [1], [7]-[10].

The general idea is to excite the aircraft using perturbation inputs with wideband frequency content over a range of frequencies that encompasses the expected modal frequencies of the aircraft dynamic response. The excitations are implemented as perturbations to the control surface deflections by summing designed perturbation inputs with the actuator commands from the pilot and feedback control system, just before the actuator limiting on command rate and position.

Each designed perturbation input is a sum of sinusoids with unique frequencies, optimized phase shifts, and a specified power distribution. Component frequencies are selected to cover a frequency band of interest, similar to frequency sweeps. The wideband frequency content of the inputs is important because there is naturally some uncertainty as to what the modal frequencies are for the aircraft in flight. Wideband inputs provide robustness to this uncertainty. Phase shifts for the sinusoidal components of each input are optimized to achieve low peak-to-peak amplitude and high input energy content for the sum of sinusoids. Amplitudes of the individual sinusoidal components can be chosen to achieve a specific power distribution to further target the input energy within the frequency band of interest.

Multiple inputs are designed to be mutually orthogonal in both the time domain and the frequency domain, and are optimized for maximum data information content in multiple axes over a short time period, while minimizing excursions from the nominal flight condition. These characteristics make the input design effective for stability and control flight testing.

Each perturbation input $\boldsymbol{u}_{j}$, which is to be applied to the $j^{\text {th }}$ individual control surface, is comprised of a set of summed harmonic sinusoids with individual phase shifts $\phi_{k}$,

$$
\boldsymbol{u}_{j}=\sum_{k \in\{1,2, \ldots, M\}} A_{k} \sin \left(\frac{2 \pi k \boldsymbol{t}}{T}+\phi_{k}\right) \quad j=1,2, \ldots, m
$$

where $M$ is the total number of available harmonically-related frequencies, $T$ is the time length of the excitation, and $A_{k}$ is the amplitude for the $k^{\text {th }}$ sinusoidal component. The variable $t$ represents a vector of $N$ discrete time points, $\boldsymbol{t}=\left[\begin{array}{lllll}t(0) & t(1) & \ldots & t(N-1)\end{array}\right]^{T}$, and $\boldsymbol{u}_{j}$ represents the vector of corresponding amplitudes for the $j^{\text {th }}$ input, $\boldsymbol{u}_{j}=\left[\begin{array}{llll}u_{j}(0) & u_{j}(1) & \ldots & u_{j}(N-1)\end{array}\right]^{T}$. Each of the $m$ inputs is comprised of selected components from the pool of $M$ harmonic sinusoids with frequencies $\omega_{k}=2 \pi k / T, k=1,2, \ldots, M$, where $\omega_{M}=2 \pi M / T$ represents the upper limit of the frequency band for the excitation. The interval $\left[\omega_{1}, \omega_{M}\right] \mathrm{rad} / \mathrm{s}$ specifies the range of frequencies where the aircraft dynamics are expected to lie. 
If the phase angles $\phi_{k}$ in Eq. (1) were chosen at random on the interval $(-\pi, \pi]$ radians, then in general, the various harmonic components would add together at some points to produce an input $\boldsymbol{u}_{j}$ with relatively large amplitude excursions. This is undesirable, because it can result in the dynamic system being moved too far from the reference condition selected for the experiment. To prevent this, the phase angles $\phi_{k}$ for the selected harmonic components are chosen to minimize relative peak factor $R P F^{1}$, defined by

$$
R P F\left(\boldsymbol{u}_{j}\right) \equiv \frac{\left[\max \left(\boldsymbol{u}_{j}\right)-\min \left(\boldsymbol{u}_{j}\right)\right] / 2}{\sqrt{2\left(\boldsymbol{u}_{j}^{T} \boldsymbol{u}_{j}\right) / N}}=\frac{\left[\max \left(\boldsymbol{u}_{j}\right)-\min \left(\boldsymbol{u}_{j}\right)\right]}{2 \sqrt{2} \operatorname{rms}\left(\boldsymbol{u}_{j}\right)}
$$

Relative peak factor is a measure of the efficiency of an input for dynamic modeling purposes, in terms of the amplitude range of the input divided by a measure of the input energy. The relative peak factor is scaled so that any individual sinusoidal component (such as any one of the summands in Eq. (1)) has $R P F=1$. Low relative peak factors are desirable and efficient for estimating dynamic model parameters, because the objective is to excite the dynamic system with sufficient input energy over a variety of frequencies while minimizing the peak-to-peak input amplitudes in the time domain, to avoid driving the dynamic system too far away from the reference condition.

For a composite signal with more than one sinusoidal component, as in Eq. (1), the goal of designing an input with minimum $R P F$ is achieved by adjusting the phase parameters $\phi_{k}$ for the sinusoidal components of the input. The resulting optimization problem is non-convex; however, a simplex algorithm ${ }^{1,11}$ can be applied to find a solution.

The integers $k$ specifying the frequencies for the $j^{\text {th }}$ input $\boldsymbol{u}_{j}$ are selected to be unique to that input, but

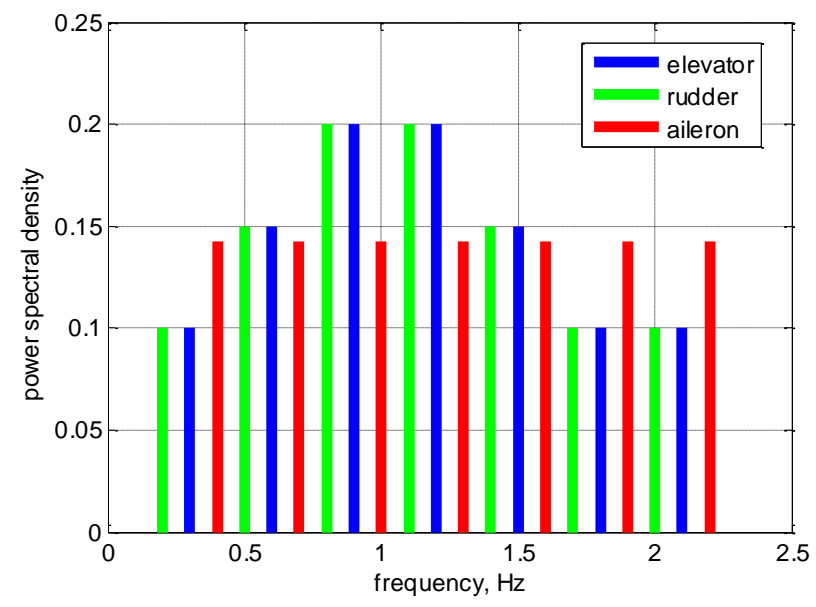

Figure 1. Multiple orthogonal phase-optimized multisine input spectra are not necessarily consecutive. A good approach for multiple inputs is to assign integers $k$ to each input alternately. This is illustrated in Figure 1 for a flight test maneuver design on the T-2 subscale jet transport aircraft. In this case, there were 3 inputs: elevator, rudder, and aileron, and a total of 21 frequencies $(M=21)$. The frequencies were interleaved among the three inputs to achieve wideband frequency content for each input. This provided robustness to uncertainty in how each control excites the dynamic modes of the aircraft. Because each input has wideband frequency content, the same input design can be applied at various flight conditions, which simplifies the flight test and reduces flight computer memory requirements.

To achieve a uniform power distribution, the $A_{k}$ are selected as

$$
A_{k}=\frac{A}{\sqrt{n}} \quad \forall k
$$

where $n$ is the number of sinusoidal components included in the summation of Eq. (1) for $\boldsymbol{u}_{j}$, and $A$ is the amplitude of the composite input $\boldsymbol{u}_{j}$. Therefore, with uniform power distribution, selection of the $A_{k}$ reduces to selecting a single value for the input amplitude $A$. Each input $\boldsymbol{u}_{j}$ can of course have arbitrary amplitude $A$, subject to practical flight testing and modeling constraints.

It is also possible to modify the power at individual frequencies for each input, to focus the excitation on frequencies near where the natural frequencies of the dynamic modes are believed to be, or to avoid exciting 
structural responses, for example. For each input, the power spectrum can be tailored by selecting the $A_{k}$ in Eq. (1) to distribute power over the spectral components. The power spectra shown in Figure 1 are normalized, so the effects of individual control surface amplitudes are excluded. This means that for each input, the sum of all the spectral line ordinates (sum of the heights of the bars for each input) is 1.

When the frequency indices $k$ selected for each input $\boldsymbol{u}_{j}$ in Eq. (1) are distinct from those chosen for the other inputs, then the frequency content of each $\boldsymbol{u}_{j}$ consists of distinct spectral lines in the frequency domain, as can be seen in Figure 1. Therefore, the vectors of Fourier transforms for the inputs as a function of frequency have inner products equal to zero. In this sense, the inputs are mutually orthogonal in the frequency domain, because each input contains frequencies that are not present in the other inputs.

In the time domain, a sum of harmonic sinusoids is orthogonal to any other sum of sinusoids with harmonicallyrelated frequencies, regardless of the constant phase shift of each sinusoidal component ${ }^{1,8}$. Consequently, the inputs are also mutually orthogonal in the time domain.

Because more than one surface is being moved at the same time, it is advantageous for modeling purposes if the $\boldsymbol{u}_{j}$ applied to the control surfaces are mutually orthogonal. In that case, the inputs are completely decorrelated, which improves the accuracy of control effectiveness estimates and reduces required data record lengths. Using the
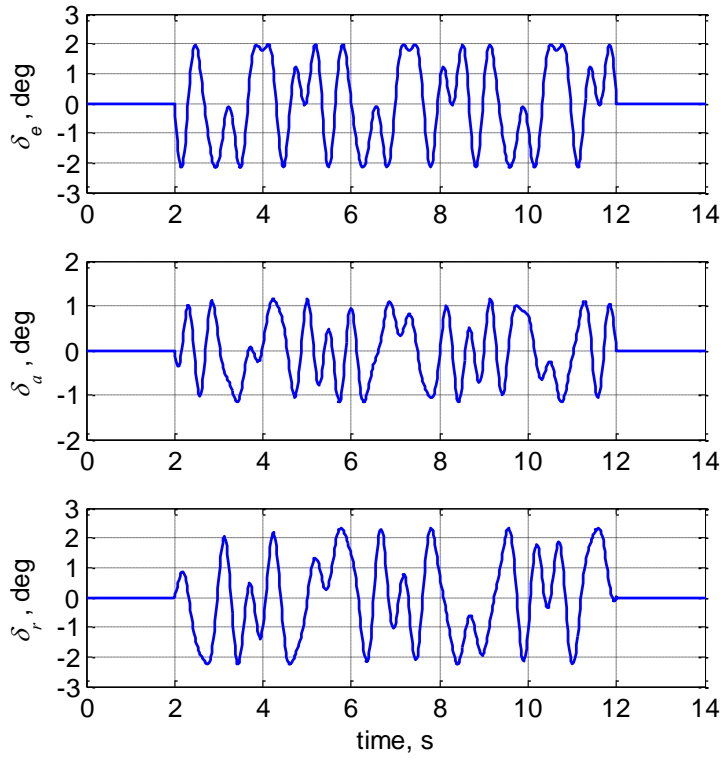

Figure 2. Multiple orthogonal phase-optimized multisine excitation inputs input design method described here, all of the $\boldsymbol{u}_{j}$ can be made mutually orthogonal in both the time and frequency domains, while also minimizing relative peak factor for each $\boldsymbol{u}_{j}$, which keeps the aircraft from departing significantly from the reference flight condition. This gives the analyst the flexibility to use either time-domain or frequency-domain modeling methods, while retaining the desirable feature of mutually orthogonal inputs.

Figure 2 shows perturbation input time series for a $10 \mathrm{~s}$ maneuver design flown on the T-2 subscale jet transport aircraft. These inputs are mutually orthogonal in both the time and frequency domains. The inputs were computed from Eq. (1) and the information in Table 1 and Figure 1, where the phase angles $\phi_{k}$ were optimized for minimum relative peak factor.

In practice, pilot inputs and feedback control (which are summed with the designed excitation inputs) can act to ruin the mutual orthogonality of the control surface deflections. However, good modeling results require only low correlations, not zero correlations, so that slightly correlated control surface deflections still work well. 


\section{Test Aircraft and Flight Data}

\section{A. T-2 Subscale Jet Transport Aircraft}

The T-2 aircraft is a 5.5 percent dynamically-scaled model of a generic commercial twin-engine jet transport aircraft. Figure 3 shows a photograph of the aircraft in flight. The aircraft has twin jet engines mounted under the wings and retractable tricycle landing gear. Aircraft geometry and nominal mass properties are given in Table 2. Further information on the T-2 subscale jet transport aircraft and associated flight test operations can be found in Refs. [12]-[14]. A similar configuration was tested extensively in the wind tunnel ${ }^{5,6}$, although the wind tunnel test articles differed in some geometric details from the aircraft used for the flight tests. The wind tunnel data

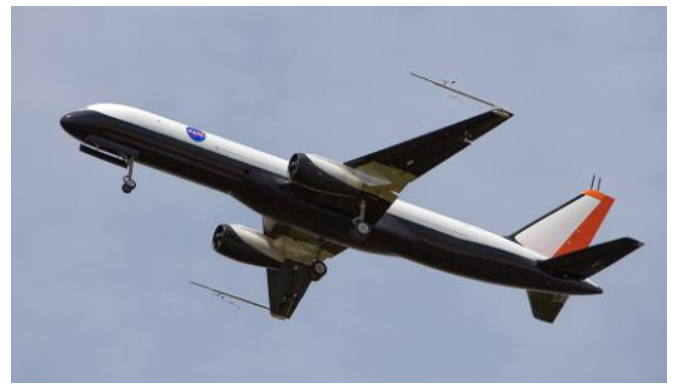

Figure 3. T-2 subscale jet transport aircraft Credit: NASA Langley Research Center provided a database for the nonlinear aircraft simulation used in this work.

Control surfaces on the T-2 aircraft are left and right ailerons, left and right inboard and outboard elevators, upper and lower rudders, left and right inboard and outboard trailing-edge flaps, and left and right inboard and outboard spoilers, for a total of 16 independent control surfaces. For the flight data analyzed in this work, only the elevators, ailerons, and rudders were deflected. The individual elevator surfaces were moved together as a single elevator surface, and similarly for the rudders. Left and right ailerons were deflected asymmetrically, in the conventional way. Definitions of control surface deflections are given below. Trailing edge down is positive deflection for wing and elevator surfaces, and trailing edge left is positive for rudder surfaces.

$$
\begin{gathered}
\delta_{e}=\frac{1}{4}\left(\delta_{e_{l o}}+\delta_{e_{l i}}+\delta_{e_{r i}}+\delta_{e_{r o}}\right) \\
\delta_{a}=\frac{1}{2}\left(\delta_{a_{r}}-\delta_{a_{l}}\right) \quad \delta_{r}=\frac{1}{2}\left(\delta_{r_{u}}+\delta_{r_{l}}\right)
\end{gathered}
$$

Flight testing was carried out at NASA Wallops Flight Facility and at Blackstone Army Airfield in Virginia, using the NASA Langley AirSTAR flight test capability. A research pilot executed the flight test maneuvers from inside a mobile control room, using a synthetic vision display drawn from telemetry data and a local terrain database, along with video from a camera in the nose of the aircraft. Inputs from the research pilot and a groundbased flight control system were used to generate control surface commands which were transmitted by telemetry to the aircraft.

The flight control system has the capability to inject automated control surface perturbations to excite the aircraft dynamic response for modeling purposes. These control surface perturbations can have arbitrary waveforms, and can be applied to multiple control surfaces individually or simultaneously. The perturbations are summed with pilot and feedback control commands in the flight control system, just before the actuator command rate and position limiting. Typically, the research pilot flies the aircraft to the desired flight condition(s), then initiates the automated control surface perturbations with a trigger switch on the control stick.

\section{B. Instrumentation and Flight Data}

The T-2 aircraft was equipped with a micro-INS, which provided 3-axis translational accelerometer measurements, angular rate measurements, estimated attitude angles, and GPS velocity and position. Air data probes attached to booms mounted on each wingtip (visible in Figure 3) measured angle of attack, sideslip angle, static pressure, and dynamic pressure. Data from these sensors were corrected for position and averaged for use in the analysis. Measurements from static pressure sensors and ambient temperature sensors were used to compute air density and altitude. Engine speeds in rpm were measured and used as inputs to an engine model to compute thrust. The engine model was identified from ground test data, with adjustments for ram drag identified from flight data. Potentiometers on the rotation axes of the control surfaces measured control surface deflections. Mass properties were computed based on measured fuel flow, pre-flight weight and balance, and body-axis inertia measurements 
done on the ground for the aircraft without fuel. Pilot stick and rudder pedal commands and throttle position were also measured and recorded. Data from onboard sensors were telemetered to the ground in real time. Sampling rate for the flight data was $200 \mathrm{~Hz}$, decimated to $50 \mathrm{~Hz}$ for data analysis and modeling.

\section{Turbulence Level Characterization Using Flight Measurements}

A simple method to quantitatively characterize atmospheric turbulence affecting the AirSTAR T-2 aircraft during flight research was developed. This was motivated by frequent adverse turbulent flight conditions resulting from requirements to operate the remotely piloted aircraft no higher than 1,500 feet above ground level (AGL). The turbulence, observed to worsen during the day, was typically attributed to convective air currents resulting from diurnal heating.

The purpose of this quantitative characterization was to efficiently improve data quality during research flights without changing the airspace limits or increasing pilot workload. To do this, the characterization method needed to be sensitive to small changes in turbulence level and be capable of being computed and updated during a flight. This capability made it possible to tailor test techniques, objectives, and methods to accommodate the in-situ turbulence level. For example, parameter estimation excitation input amplitudes were scaled in real time based on the quantified turbulence characteristics.

\section{A. Method}

Convection due to diurnal heating was assumed to be the most prevalent physical process creating turbulence for the T-2 aircraft. Thus, the body-axis vertical component of airspeed was assumed to be the airplane response variable with the most sensitivity to turbulence effects and the standard deviation of that signal would provide a useful characterization of turbulence. Vertical component of airspeed in the aircraft body-axis system was calculated from angle of attack and airspeed measurements at a $50 \mathrm{~Hz}$ rate. The power spectral density of this signal was calculated using the SIDPAC spectral analysis function spect.m, which employs a frequency binning approach $^{1}$. In the computation of the standard deviation of this signal, only the power in the $2 \mathrm{~Hz}$ to $7 \mathrm{~Hz}$ range was used. The purpose of neglecting frequencies below $2 \mathrm{~Hz}$ was to avoid contamination of the turbulence characterization with effects from commanded dynamics associated with routine maneuvering, flight test techniques, and parameter estimation excitation inputs. For computational efficiency, frequencies above $7 \mathrm{~Hz}$ were not used because there was not significant power in that frequency range. The resulting standard deviation of the vertical velocity component in the 2 to $7 \mathrm{~Hz}$ range $\left(\sigma_{\mathrm{w}[2-7 \mathrm{~Hz}]}\right)$ was assumed to be predominantly due to turbulence. All structural modes of the T-2 aircraft are above $10 \mathrm{~Hz}$.

\section{B. Correlation with Pilot Comments}

To establish confidence in the methodology, turbulence level was quantified for a series of flights and compared to pilot subjective turbulence ratings provided during those flights. For this analysis, the metric, $\sigma_{\mathrm{w}[2-7 \mathrm{~Hz}]}$, was computed at 1 second intervals based on a sliding 30-second window of data. This approach allowed in-flight turbulence variation to be automatically tracked. Because the flights were all short (approximately 15 minutes) and in general had little temporal or altitude variation, the mean value of $\sigma_{\mathrm{w}[2-7 \mathrm{~Hz}]}$ for the entire research segment of the flight was used to compare with pilot comments. Very good agreement and sensitivity is shown in Figure 4, which illustrates the correlation of the turbulence characterization metric with the pilot's subjective turbulence rating. Use of alternate response variables such as normal acceleration, lateral acceleration, and sideslip angle were also investigated. However, the vertical velocity component derived from angle of attack provided the best and most consistent agreement with pilot ratings. The vertical velocity component has the advantage of high sensitivity and a more direct relationship to turbulence, as opposed to body-axis accelerations, for example, which represent aircraft response to turbulence, or air flow angles, which are less sensitive because they involve (approximately) division by airspeed. The lines indicating light, moderate, and severe turbulence in Figure 4 were selected to represent the best correlation of the quantitative turbulence metric with the pilot's subjective rating of the turbulence level.

Figures 5 and 6 illustrate a typical and an atypical turbulence profile, respectively. The turbulence level for the flight depicted in Figure 5 was rated by the pilot as "light" turbulence. Good agreement is seen with the turbulence metric distributed around a mean of $0.28 \mathrm{ft} / \mathrm{s}$. By comparison, Figure 6 shows an unusually abrupt variation of the turbulence metric with altitude. During this flight profile, which included a stall series, the pilot commented that at 1,400 feet AGL the turbulence was "very light to light", but between 900 and 1,200 feet the turbulence was "moderate to severe". Good agreement is seen, as the turbulence characteristics were captured by the turbulence metric as altitude varied during the stall series. 
Figure 7 shows the average turbulence metric (markers) and standard error (vertical bars) for all 58 flights to date of the T-2 aircraft. The first 12 flights were at NASA Wallops, and the last 46 flights were at Blackstone Army Airfield. The markers indicate the mean value of the turbulence metric computed over the entire flight, and the vertical bars show \pm 2 standard deviations. This data was used extensively to develop and evaluate the analytic method for dynamic modeling in turbulence described next.

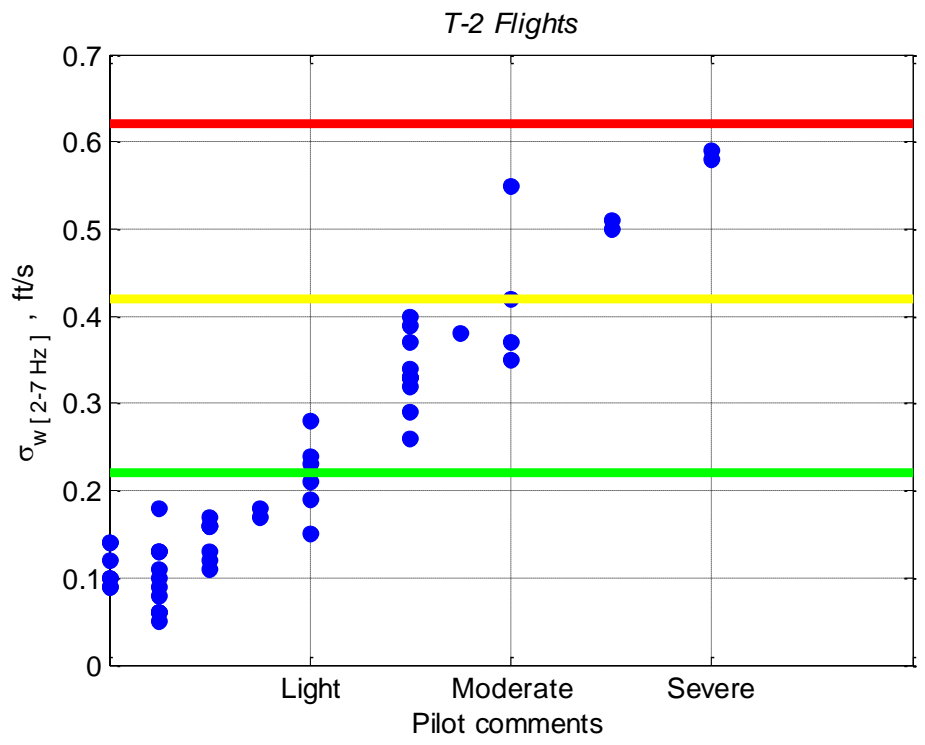

Figure 4. Plot of turbulence characterization metric versus pilot turbulence ratings

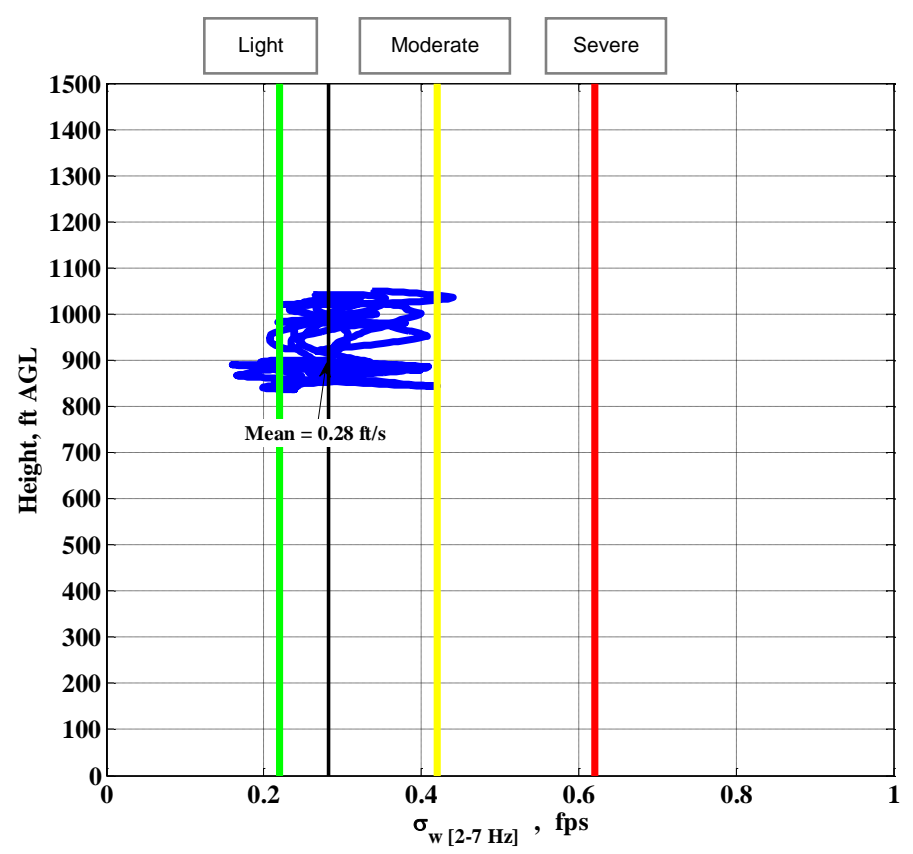

Figure 5. Typical turbulence characterization metric during a flight where the pilot rated the turbulence as "light" 


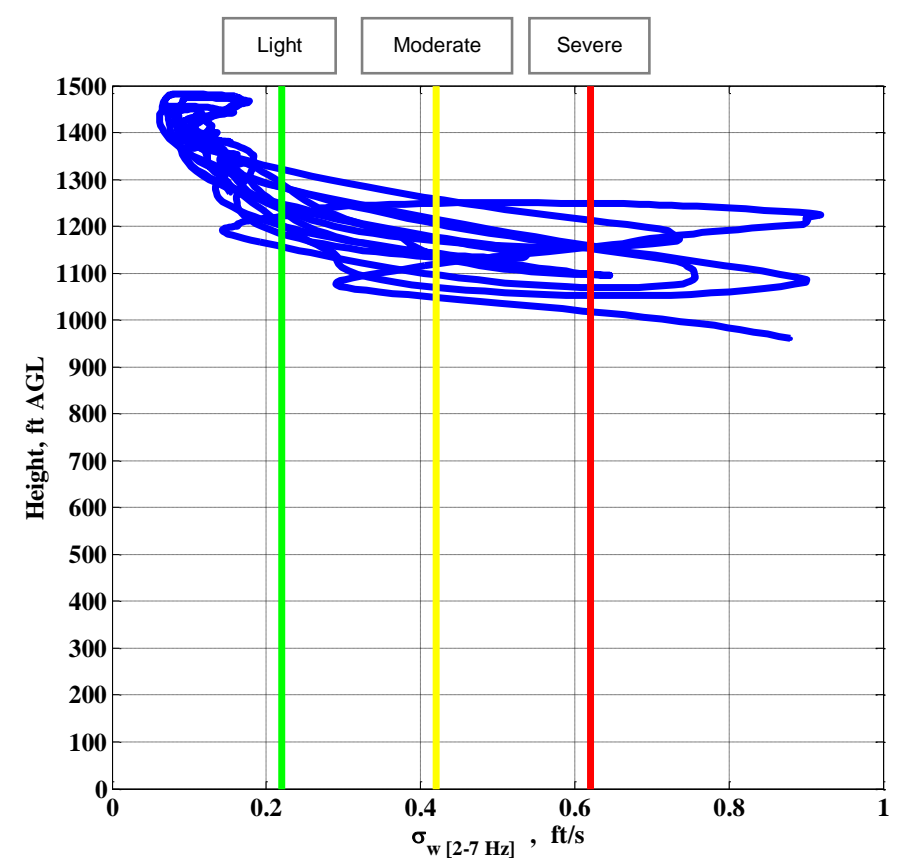

Figure 6. Turbulence characterization metric captures significant increase in turbulence (moderate to severe) during a series of climbs and descents

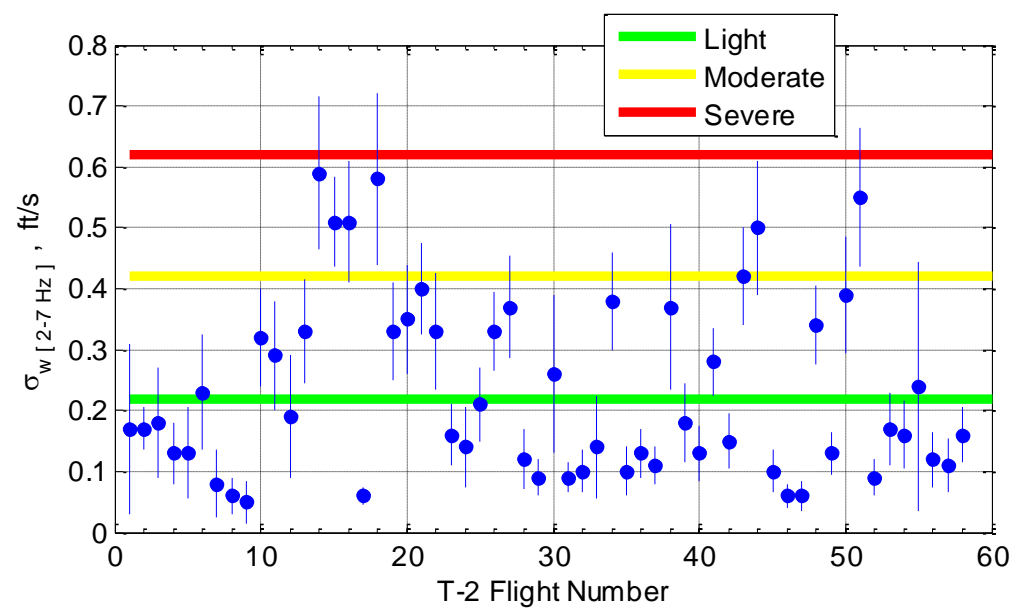

Figure 7. Turbulence metric for T-2 flights

\section{Dynamic Modeling in Turbulence}

\section{A. Aircraft Dynamic Modeling}

For aircraft dynamic modeling from flight data, the unknown model parameters in the aircraft equations of motion are typically nondimensional stability and control derivatives, which are influence coefficients for the aircraft states and controls on the nondimensional aerodynamic force and moment coefficients. The modeling problem can be partitioned for each force or moment coefficient, corresponding to minimizing the equation error in each individual equation of motion for the six rigid-body degrees of freedom of the aircraft. In this formulation, the nondimensional force and moment coefficients are treated as the response variables to be modeled in terms of aircraft states and controls. 
Values for the nondimensional aerodynamic force and moment coefficients cannot be measured directly in flight, but instead must be computed from measured and known quantities using the following equations ${ }^{1}$

$$
\begin{gathered}
C_{X}=\left(m a_{x}-T_{x}\right) / \bar{q} S \\
C_{Z}=\left(m a_{z}-T_{z}\right) / \bar{q} S \\
C_{m}=\left[I_{y} \dot{q}+\left(I_{x}-I_{z}\right) p r+I_{x z}\left(p^{2}-r^{2}\right)-M_{T}\right] / \bar{q} S \bar{c} \\
C_{Y}=m a_{y} / \bar{q} S \\
C_{l}=\left[I_{x} \dot{p}-I_{x z}(p q+\dot{r})+\left(I_{z}-I_{y}\right) q r\right] / \bar{q} S b \\
C_{n}=\left[I_{z} \dot{r}-I_{x z}(\dot{p}-q r)+\left(I_{y}-I_{x}\right) p q\right] / \bar{q} S b
\end{gathered}
$$

These expressions retain the full nonlinear dynamics in the equations of motion for a symmetric rigid aircraft. For local modeling over a short time period, the force and moment coefficients computed from Eqs. (6) and (7) can be modeled using linear expansions in the aircraft states and controls:

$$
\begin{gathered}
C_{X}=C_{X_{\alpha}} \Delta \alpha+C_{X_{q}} \frac{\Delta q \bar{c}}{2 V}+C_{X_{\delta}} \Delta \delta+C_{X_{o}} \\
C_{Z}=C_{Z_{\alpha}} \Delta \alpha+C_{Z_{q}} \frac{\Delta q \bar{c}}{2 V}+C_{Z_{\delta}} \Delta \delta+C_{Z_{o}} \\
C_{m}=C_{m_{\alpha}} \Delta \alpha+C_{m_{q}} \frac{\Delta q \bar{c}}{2 V}+C_{m_{\delta}} \Delta \delta+C_{m_{o}} \\
C_{Y}=C_{Y_{\beta}} \Delta \beta+C_{Y_{p}} \frac{\Delta p b}{2 V}+C_{Y_{r}} \frac{\Delta r b}{2 V}+C_{Y_{\delta}} \Delta \delta+C_{Y_{o}} \\
C_{l}=C_{l_{\beta}} \Delta \beta+C_{l_{p}} \frac{\Delta p b}{2 V}+C_{l_{r}} \frac{\Delta r b}{2 V}+C_{l_{\delta}} \Delta \delta+C_{l_{o}} \\
C_{n}=C_{n_{\beta}} \Delta \beta+C_{n_{p}} \frac{\Delta p b}{2 V}+C_{n_{r}} \frac{\Delta r b}{2 V}+C_{n_{\delta}} \Delta \delta+C_{n_{o}}
\end{gathered}
$$

The $\Delta$ notation indicates perturbation from a reference condition. In each expansion, a single term is shown to represent all relevant and similar control terms, to simplify the expressions. For example, in Eq. (9b), the term $C_{l_{\delta}} \Delta \delta$ represents all the control terms for $C_{l}$, e.g., $C_{l_{\delta}} \Delta \delta \equiv C_{l_{\delta_{a}}} \Delta \delta_{a}+C_{l_{\delta_{r}}} \Delta \delta_{r}$. In Eq. (9b), $C_{l_{o}}$ represents the steady-state nondimensional rolling moment at a reference condition, and similarly for the other expansions.

The linear aerodynamic models in Eqs. (8) and (9) contain parameters called stability and control derivatives, such as $C_{l_{\beta}}$ and $C_{l_{\delta_{a}}}$, that characterize the stability and control of the aircraft. For short periods of time, the stability and control derivatives are considered as constant model parameters to be estimated from flight data.

The next subsection describes how the unknown stability and control derivatives in the linear aerodynamic models of Eqs. (8) and (9) can be estimated from flight data using equation-error parameter estimation in the frequency domain. 


\section{B. Stability and Control Derivative Estimation in the Frequency Domain}

The first step required for modeling in the frequency domain is to transform the measured flight data from the time domain into the frequency domain. The finite Fourier transform is the analytical tool used for this task. For an arbitrary scalar signal $x(t)$ on the time interval $[0, T]$, the finite Fourier transform is defined by

$$
\mathcal{F}[x(t)] \equiv \tilde{x}(\omega) \equiv \int_{0}^{T} x(t) e^{-j \omega t} d t
$$

which can be approximated by

$$
\tilde{x}(\omega) \approx \Delta t \sum_{i=0}^{N-1} x(i) e^{-j \omega i \Delta t}
$$

where $x(i) \equiv x(i \Delta t), T=(N-1) \Delta t$, and $\Delta t$ is a constant sampling interval. The summation in Eq. (11) is defined as the discrete Fourier transform,

$$
X(\omega) \equiv \sum_{i=0}^{N-1} x(i) e^{-j \omega i \Delta t}
$$

so that the finite Fourier transform approximation can be written as

$$
\tilde{x}(\omega) \approx X(\omega) \Delta t
$$

Some fairly straightforward corrections ${ }^{1}$ can be made to remove the inaccuracy resulting from the fact that Eq. (12b) is a simple Euler approximation to the finite Fourier transform in Eq. (10). However, if the sampling rate is much higher than the frequencies of interest, as is typically the case for dynamic modeling from flight data, then the corrections are small and can be safely ignored.

For each aerodynamic model in Eqs. (8) and (9), the parameter estimation problem can be formulated in the frequency domain as a standard least squares regression problem with complex data ${ }^{1}$,

$$
\tilde{\boldsymbol{z}}=\tilde{\boldsymbol{X}} \boldsymbol{\theta}+\tilde{\boldsymbol{e}}
$$

where, for example, using the rolling moment equation (9b),

$$
\begin{aligned}
& \tilde{z}=\left[\begin{array}{llll}
\tilde{C}_{l}(1) & \tilde{C}_{l}(2) & \ldots & \tilde{C}_{l}(M)
\end{array}\right]^{T} \\
& \tilde{\boldsymbol{X}}=\left[\begin{array}{ccccc}
\Delta \tilde{\beta}(1) & \Delta \tilde{p}_{n}(1) & \Delta \tilde{r}_{n}(1) & \Delta \tilde{\delta}_{a}(1) & \Delta \tilde{\delta}_{r}(1) \\
\Delta \tilde{\beta}(2) & \Delta \tilde{p}_{n}(2) & \Delta \tilde{r}_{n}(2) & \Delta \tilde{\delta}_{a}(2) & \Delta \tilde{\delta}_{r}(2) \\
\vdots & \vdots & \vdots & \vdots & \vdots \\
\Delta \tilde{\beta}(M) & \Delta \tilde{p}_{n}(M) & \Delta \tilde{r}_{n}(M) & \Delta \tilde{\delta}_{a}(M) & \Delta \tilde{\delta}_{r}(M)
\end{array}\right] \\
& \boldsymbol{\theta}=\left[\begin{array}{lllll}
C_{l_{\beta}} & C_{l_{p}} & C_{l_{r}} & C_{l_{\delta_{a}}} & C_{l_{\delta_{r}}}
\end{array}\right]^{T}
\end{aligned}
$$


The notation $\Delta \tilde{p}_{n}$ represents $\mathcal{F}[p b / 2 V]$, and similarly for $\Delta \tilde{r}_{n}$, and $\tilde{\boldsymbol{e}}$ represents the complex vector of equation errors in the frequency domain. The symbols $\Delta \tilde{\beta}(k), k=1,2, \ldots, M$ denote the Fourier transform of the sideslip angle perturbation for each frequency $\omega_{k}$, and similarly for other quantities. Each transformed variable depends on frequency. The frequencies $\omega_{k}$ can be chosen arbitrarily, and are therefore chosen to cover the frequency band where the aircraft dynamics lie. The least squares cost function is

$$
J(\theta)=\frac{1}{2}(\tilde{z}-\tilde{\boldsymbol{X}} \boldsymbol{\theta})^{\dagger}(\tilde{z}-\tilde{\boldsymbol{X}} \boldsymbol{\theta})
$$

This cost function contains $M$ squared-error terms in summation, corresponding to $M$ frequencies of interest. Similar cost expressions can be written for individual lines from Eqs. (8) and (9). The parameter vector estimate that minimizes the least squares cost function is computed from ${ }^{1}$

$$
\hat{\boldsymbol{\theta}}=\left[\operatorname{Re}\left(\tilde{\boldsymbol{X}}^{\dagger} \tilde{\boldsymbol{X}}\right)\right]^{-1} \operatorname{Re}\left(\tilde{\boldsymbol{X}}^{\dagger} \tilde{z}\right)
$$

The estimated parameter covariance matrix is ${ }^{1}$

$$
\boldsymbol{\Sigma}(\hat{\boldsymbol{\theta}}) \equiv E\left[(\hat{\boldsymbol{\theta}}-\boldsymbol{\theta})(\hat{\boldsymbol{\theta}}-\boldsymbol{\theta})^{T}\right]=\sigma^{2}\left[\operatorname{Re}\left(\tilde{\boldsymbol{X}}^{\dagger} \tilde{\boldsymbol{X}}\right)\right]^{-1}
$$

where the equation-error variance $\sigma^{2}$ can be estimated from the residuals,

$$
\hat{\sigma}^{2}=\frac{1}{\left(M-n_{p}\right)}\left[(\tilde{z}-\tilde{\boldsymbol{X}} \hat{\boldsymbol{\theta}})^{\dagger}(\tilde{z}-\tilde{\boldsymbol{X}} \hat{\boldsymbol{\theta}})\right]
$$

and $n_{p}$ is the number of unknown parameters, i.e., the number of elements in parameter vector $\boldsymbol{\theta}$. Estimated parameter standard errors are computed as the square root of the diagonal elements of the $\Sigma(\hat{\theta})$ matrix from Eq. (16), using $\hat{\sigma}^{2}$ from Eq. (17). Explanations of why the estimated parameter standard errors are computed in this way, and why this calculation in the frequency domain produces parameter error measures that are consistent with the scatter in parameter estimates from repeated maneuvers, can be found in Ref. [1]. Realistic simulation testing and flight test data analysis have shown that the accuracy of model parameters estimated with this method is comparable to using a time-domain output-error method employing iterative nonlinear optimization ${ }^{15}$.

\section{Real-Time Application}

Equation-error aerodynamic modeling in the frequency domain, described in the previous section, can be implemented in real time using a recursive Fourier transform. For a given frequency $\omega$, the discrete Fourier transform in Eq. (12a) at time $i \Delta t$, denoted by $X_{i}(\omega)$, is related to the discrete Fourier transform at time $(i-1) \Delta t$ by

$$
X_{i}(\omega)=X_{i-1}(\omega)+x(i) e^{-j \omega i \Delta t}
$$

where

$$
e^{-j \omega i \Delta t}=e^{-j \omega \Delta t} e^{-j \omega(i-1) \Delta t}
$$


The quantity $e^{-j \omega \Delta t}$ is constant for a given frequency $\omega$ and constant sampling interval $\Delta t$. It follows that the discrete Fourier transform can be computed for a given frequency at each time step using one addition in Eq. (18) and two multiplications - one in Eq. (19) using the stored constant $e^{-j \omega \Delta t}$ for frequency $\omega$, and one in Eq. (18). There is no need to store the time-domain data in memory when computing the discrete Fourier transform in this way, because the data for each sample time is processed immediately. Time-domain data from the past can be used in all subsequent analysis by simply continuing the recursive calculation of the Fourier transform. In this sense, the recursive Fourier transform acts as memory for the information in the data. More data from more maneuvers improves the quality of the data in the frequency domain without increasing memory requirements to store it. Furthermore, the Fourier transform is available at any time $i \Delta t$. The approximation to the finite Fourier transform is completed using Eq. (12b).

Note that the recursive Fourier transform update need not be done for every sampled time point. Systematically skipping time points effectively lowers the sampling rate of the data prior to Fourier transformation. This saves computation, and, assuming proper analog anti-alias filtering ${ }^{1}$ has been implemented in the instrumentation system, does not have a significant adverse impact on the parameter estimation results until the Fourier transform update rate gets below approximately 5 times the highest frequency of interest for the dynamic system. The parameter estimation calculations in Eqs. (15)-(17) are applied to frequency-domain data from the recursive Fourier transform at selected times, normally at regular time intervals, such as $0.5 \mathrm{~s}$, which corresponds to an update rate of $2 \mathrm{~Hz}$. Other values could be chosen for this time interval, but linearized aerodynamic characteristics can normally be captured very well by updating the parameter estimates at a rate of 1 or $2 \mathrm{~Hz}$, except in cases of strong nonlinearity, damage, failure, or rapid maneuvering. For those situations, the update rate can be increased, at the cost of more computations.

Estimated parameter standard errors computed from the covariance matrix in the frequency domain using Eqs. (16) and (17) do not require correction for colored residuals ${ }^{1,15}$. These standard errors are therefore a good representation of the uncertainty in the estimated parameters.

Detailed discussion of the practical aspects and advantages of aerodynamic modeling in real time using this approach can be found in Refs. [1], [9]-[10]. Note that the turbulence metric based on the vertical component of airspeed could be computed in real time using the recursive Fourier transform with the proper scaling to compute power spectral density, where the frequencies of the Fourier transform would be selected in the range [2, 7] Hz.

\section{Modifications for Aircraft Dynamic Modeling in Turbulence}

The approach to dynamic modeling in turbulence developed here could be described as mathematically minimizing the effect of turbulence, as opposed to incorporating the turbulence into the dynamic modeling. The effects of turbulence on aircraft response measurements are similar to colored noise, in that the frequency content is generally wide-band, with power concentrated at lower frequencies near the frequencies of the aircraft rigid-body dynamic modes. It is this latter characteristic that causes problems for dynamic modeling, because atmospheric turbulence is an unmeasured excitation input in a frequency range that causes significant aircraft dynamic response. When the turbulence is not measured or included mathematically in the model, any aircraft response due to the turbulence is force-fitted by parameter estimation algorithms, using other explanatory variables that happen to have nearly the right characteristics to at least partly explain the effects of turbulence. This phenomenon is the source of parameter estimation errors for dynamic modeling in turbulence. The problem is universal to all parameter estimation routines based on least squares fitting, not just equation-error in the frequency domain.

One way to reduce the effects of turbulence on the least-squares parameter estimates is to simply increase the amplitude of the excitation inputs. In the frequency domain, this raises the amplitudes associated with the excitation higher above the wide-band noise and turbulence, enhancing signal-to-noise ratio for the parameter estimation problem and improving the accuracy of the dynamic modeling results. Least-squares estimation will naturally try to fit larger peaks in the data preferentially, so that as the peaks in the frequency-domain data at the excitation frequencies increase, less attention is given to the frequency content from noise and turbulence. However, there are practical limits to this approach, because larger excitation amplitudes can evoke dynamic and aerodynamic nonlinearities, and there is the negative practical consequence that increasing turbulence levels require higher excitation amplitudes, making for potentially violent maneuvers. This approach might be described as dominating the turbulence by brute force in the experiment design, and it can work well for low turbulence levels.

As an alternative, an analytical approach can be used to address the problem. When the flight test maneuvers are implemented using orthogonal optimized multisine inputs, as described in Section II., the aircraft dynamic response 
consists of small amplitude motion about a reference condition. In this case, the dynamic modeling can be done in real time using a linear model structure and frequency-domain methods, as discussed earlier. In the absence of turbulence, the aircraft dynamic response will be comprised predominantly of components related to the designed input excitation frequencies. Because the frequency content of the excitation inputs is known from the input design process, and because the multiple input excitations are mutually orthogonal, the predominant dynamic response of the aircraft to those known excitation inputs has a known form or model structure in the frequency domain. This idea can be used in conjunction with frequency-domain data and least squares fitting to effectively remove the effects of turbulence from the explanatory variables. Stated another way, the idea is to extract or sift the portion of the frequency-domain data for each explanatory variable that resulted directly from the known designed input excitation. Everything else is from some other source, such as turbulence or measurement noise, and should be discarded.

Consider a time series consisting of a simple sinusoidal signal $x$ at a single frequency,

$$
x=A_{k} \sin \left(\omega_{k} t+\phi_{k}\right)
$$

The finite Fourier transform at frequency $\omega_{k}$ for a time period $T$ equal to an integer number of periods $T=n T_{k}$ where $T_{k}=2 \pi / \omega_{k}$ is

$$
\tilde{x}\left(\omega_{k}\right)=\int_{0}^{n T_{k}} A_{k} \sin \left(\omega_{k} t+\phi_{k}\right) e^{-j \omega_{k} t} d t=\frac{n T_{k}}{2} A_{k} e^{-j \phi_{k}}
$$

In practice, the data record can have arbitrary length $T$, which causes frequency content at $\omega_{k}$ to be smeared to neighboring frequencies by the finite Fourier transform. This is called leakage. Furthermore, analysis in the frequency domain is normally conducted at many different frequencies, not just $\omega_{k}$.

Applying the finite Fourier transform to the simple sinusoidal signal $x$ with frequency content at $\omega_{k}$ for an arbitrary record length $T$ and an arbitrary transform frequency $\omega$,

$$
\tilde{x}(\omega)=\int_{0}^{T} A_{k} \sin \left(\omega_{k} t+\phi_{k}\right) e^{-j \omega t} d t=\int_{0}^{T} A_{k} \frac{e^{j\left(\omega_{k} t+\phi_{k}\right)}-e^{-j\left(\omega_{k} t+\phi_{k}\right)}}{2 j} e^{-j \omega t} d t
$$

This integral can be computed analytically as

$$
\begin{gathered}
\tilde{x}(\omega)=A_{k}\left\{\left[\frac{e^{-j\left(\omega-\omega_{k}\right) T}-1}{2\left(\omega-\omega_{k}\right)}\right] e^{j \phi_{k}}+\left[\frac{1-e^{-j\left(\omega+\omega_{k}\right) T}}{2\left(\omega+\omega_{k}\right)}\right] e^{-j \phi_{k}}\right\} \\
\tilde{x}(\omega)=A_{k}\left\{e^{-j\left(\omega-\omega_{k}\right) T / 2}\left[\frac{e^{-j\left(\omega-\omega_{k}\right) T / 2}-e^{j\left(\omega-\omega_{k}\right) T / 2}}{2\left(\omega-\omega_{k}\right)}\right] e^{j \phi_{k}}+e^{-j\left(\omega+\omega_{k}\right) T / 2}\left[\frac{e^{j\left(\omega+\omega_{k}\right) T / 2}-e^{-j\left(\omega+\omega_{k}\right) T / 2}}{2\left(\omega+\omega_{k}\right)}\right] e^{-j \phi_{k}}\right\} \\
\tilde{x}(\omega)=A_{k}\left\{-j \frac{T}{2} e^{-j\left(\omega-\omega_{k}\right) T / 2} \operatorname{sinc}\left[\left(\omega-\omega_{k}\right) T / 2\right] e^{j \phi_{k}}+j \frac{T}{2} e^{-j\left(\omega+\omega_{k}\right) T / 2} \operatorname{sinc}\left[\left(\omega+\omega_{k}\right) T / 2\right] e^{-j \phi_{k}}\right\}
\end{gathered}
$$

where $\sin c(x) \equiv \sin (x) / x$ and $\sin c(0)=1$. Re-writing the last equation, 


$$
\begin{aligned}
\tilde{x}(\omega)= & A_{k} \sin \left(\phi_{k}\right) \frac{T}{2}\left\{e^{-j\left(\omega-\omega_{k}\right) T / 2} \operatorname{sinc}\left[\left(\omega-\omega_{k}\right) T / 2\right]+e^{-j\left(\omega+\omega_{k}\right) T / 2} \operatorname{sinc}\left[\left(\omega+\omega_{k}\right) T / 2\right]\right\} \\
& +A_{k} \cos \left(\phi_{k}\right) j \frac{T}{2}\left\{-e^{-j\left(\omega-\omega_{k}\right) T / 2} \operatorname{sinc}\left[\left(\omega-\omega_{k}\right) T / 2\right]+e^{-j\left(\omega+\omega_{k}\right) T / 2} \operatorname{sinc}\left[\left(\omega+\omega_{k}\right) T / 2\right]\right\}
\end{aligned}
$$

Now define

$$
\begin{aligned}
& a \equiv A_{k} \sin \left(\phi_{k}\right) \\
& b \equiv A_{k} \cos \left(\phi_{k}\right)
\end{aligned}
$$

so that

$$
\begin{aligned}
\tilde{x}(\omega)= & a \frac{T}{2}\left\{e^{-j\left(\omega-\omega_{k}\right) T / 2} \operatorname{sinc}\left[\left(\omega-\omega_{k}\right) T / 2\right]+e^{-j\left(\omega+\omega_{k}\right) T / 2} \operatorname{sinc}\left[\left(\omega+\omega_{k}\right) T / 2\right]\right\} \\
& +b j \frac{T}{2}\left\{-e^{-j\left(\omega-\omega_{k}\right) T / 2} \operatorname{sinc}\left[\left(\omega-\omega_{k}\right) T / 2\right]+e^{-j\left(\omega+\omega_{k}\right) T / 2} \operatorname{sinc}\left[\left(\omega+\omega_{k}\right) T / 2\right]\right\}
\end{aligned}
$$

The quantities multiplying $a$ and $b$, which can be called frequency-domain leakage functions, depend only on the frequency $\omega_{k}$, the transform frequency $\omega$, and the data record length $T$. Parameters $a$ and $b$ contain information on magnitude and phase of the finite Fourier transform. These quantities together constitute all of the information needed to compute $\tilde{x}(\omega)$ analytically. The finite Fourier transform of $x(t)$ is a linear combination of the quantities multiplying the parameters $a$ and $b$ in the above equation.

The quantities multiplying $a$ and $b$ in Eq. (24) can also be interpreted as basis functions for the frequency domain data for sinusoidal signals with frequency $\omega_{k}$, computed at the transform frequencies $\omega$, when the data record length $T$ is arbitrary. The finite Fourier transform of a multisine input would be a sum of terms like those in Eq. (24), for each $\omega_{k}$. Because of linearity and the discrete frequencies used in the multisine excitation inputs, aircraft responses to those excitation inputs in the steady state are known to be comprised of linear combinations of sinusoids at the specific frequencies $\omega_{k}$ contained in the multisine excitation inputs. Using that information in combination with the known form of the frequency-domain leakage functions derived above, the steady state aircraft response to the excitation inputs can be modeled in the frequency domain using the frequency leakage functions computed from the above equation, with unknown parameters $a$ and $b$ for each known excitation frequency $\omega_{k}$. If each aircraft response is modeled in this way, using basis functions that represent the frequency content expected from the input excitations only, the aircraft response from turbulence will be treated as extraneous noise, and will be mostly discarded. In effect, the known form of the aircraft response to designed excitation inputs in the frequency domain is used to separate the aircraft dynamic response to designed excitation inputs from the total aircraft response, which includes aircraft response to turbulence. This process might be called sifting the explanatory variable data in the frequency domain.

To use this approach, each aircraft response used as an explanatory variable in the aerodynamic modeling (e.g., air flow angles and body-axis angular rates) must be first modeled in the frequency domain using frequency leakage basis functions computed from Eq. (24). The modeled explanatory variable data then replaces the measured explanatory data in the frequency domain, and the modeling can proceed as usual, in the manner described earlier in Sections V.A. and V.B. This data conditioning step in the frequency domain is used to remove the parts of the aircraft response due to turbulence (and other sources, such as measurement noise), so that the dynamic modeling can be done accurately using conventional and previously-validated equation-error parameter estimation methods in the frequency domain. Results can be improved further if the measured outputs, such as nondimensional force and moment coefficients, are also sifted in the frequency domain in the same manner, prior to the modeling. 
Note that the sifting technique is really only valid for the steady-state part of the aircraft response. Consequently, transient response introduces error into the sifting process and consequently also into the parameter estimation based on sifted frequency-domain data. Using longer time periods of excitation or omitting the first few seconds of the data record after the excitation begins should improve the parameter estimation results using the sifting technique.

\section{Simulation Results}

The sifting technique in the frequency domain was applied in simulation to a multisine signal corrupted with colored noise. The multisine signal had seven sinusoidal components with randomly selected amplitudes, frequencies, and phase angles. The colored noise was sized to produce a low signal-to-noise ratio of 2.5 , with 80
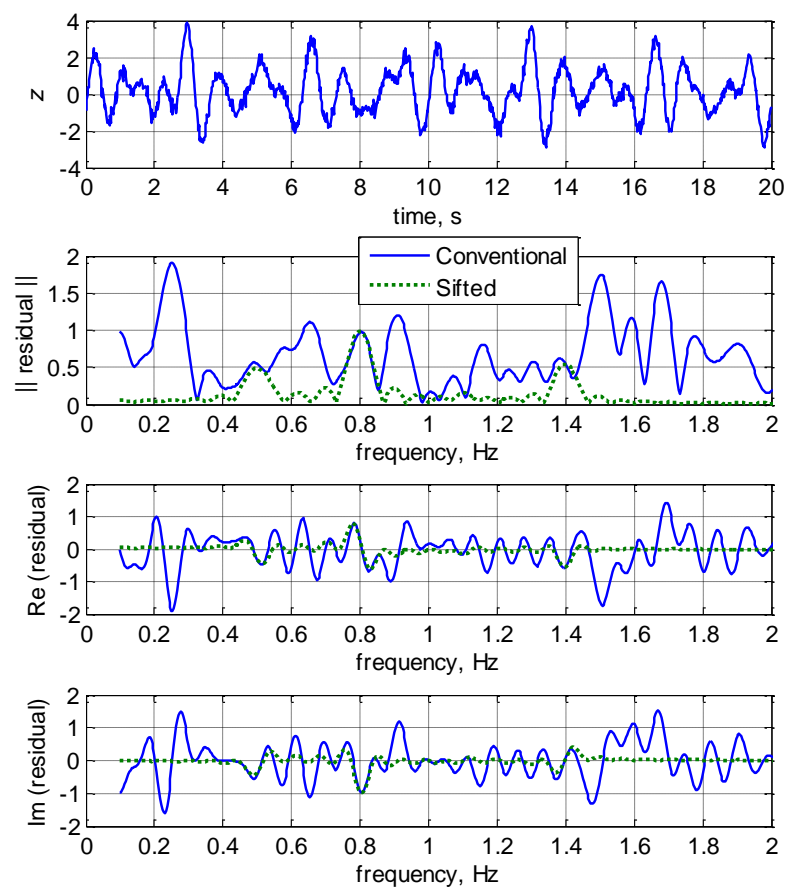

Figure 8. Accuracy of the sifting process in the frequency domain for a simulated multisine time series with turbulence

percent of the noise power confined to the same frequency range as the multisine frequencies, namely $[0,2] \mathrm{Hz}$. This simulates an aircraft linear response (such as angle of attack) to a multisine input excitation, measured in severe turbulence. The top plot in Figure 8 shows the simulated measured time series. The lower three plots in Figure 8 show the difference (residual) between the frequency-domain data for the noisefree signal and frequency-domain data obtained by applying both the conventional finite Fourier transform of Eq. (11) and the sifting process described in Section V.D. to the simulated noisy data. The transform frequencies were uniformly spaced at $0.067 \mathrm{~Hz}$ intervals over the frequency range $[0.1,2] \mathrm{Hz}$. The data window analyzed was selected as the time interval $[0,15] \mathrm{s}$. These selections were made arbitrarily, but with the intent to closely simulate flight data. The lower three plots in Figure 8 show the complex frequency-domain residuals in terms of magnitude, real part, and imaginary part. These plots represent the true errors, and show clearly that the sifting procedure produces a much more accurate representation of the frequency content of the noise-free multisine signal, compared to using a conventional finite Fourier transform. This test was repeated many times using different noise realizations, with the result that the sifting process routinely resulted in a factor of 2 to 4 reduction in the magnitude of the residual, compared to using the conventional finite Fourier transform. For the data shown in Figure 8, the magnitude of the residual was reduced by a factor of 3.38 using the sifting process. The sifting process provides a significant improvement in the accuracy of the frequency domain data for a multisine signal corrupted by colored noise. Note that the sifting process is not perfect, because frequency-domain data from the conventional finite Fourier transform applied to the noisy data is used as the measured output to be modeled in terms of frequency-domain leakage functions computed from Eq. (24). Removing noise and turbulence components from the frequency-domain data for an explanatory variable will result in more accurate model parameter estimates, reducing both bias errors and random errors ${ }^{1,15}$.

The techniques for flight test maneuver design and dynamic modeling in turbulence were then applied to a linear dynamic model of the T-2 subscale jet transport aircraft. Studying dynamic modeling techniques in simulation allows comparison of the aerodynamic model parameters identified from simulated data with the known parameters in the underlying linear dynamic model used for the simulation. Linearizing the T-2 aircraft nonlinear simulation using central finite differences at a nominal flight condition of $4 \mathrm{deg}$ angle of attack and $1500 \mathrm{ft}$ altitude resulted in the following linear dynamic model for the T-2 longitudinal short period dynamics: 


$$
\begin{gathered}
{\left[\begin{array}{c}
\dot{\alpha} \\
\dot{q}
\end{array}\right]=\left[\begin{array}{cc}
-2.59 & 0.942 \\
-37.4 & -3.36
\end{array}\right]\left[\begin{array}{l}
\alpha \\
q
\end{array}\right]+\left[\begin{array}{l}
-0.005 \\
-0.702
\end{array}\right] \delta_{e}} \\
{\left[\begin{array}{c}
\alpha \\
q \\
a_{z}
\end{array}\right]=\left[\begin{array}{cc}
1 & 0 \\
0 & 1 \\
-10.2 & -0.226
\end{array}\right]\left[\begin{array}{l}
\alpha \\
q
\end{array}\right]+\left[\begin{array}{c}
0 \\
0 \\
-0.018
\end{array}\right] \delta_{e}}
\end{gathered}
$$

Simulated aircraft responses were corrupted with white Gaussian noise scaled so that root-mean-square wide-band noise amplitudes were similar to what was observed in T-2 flight data. Along with the wide-band noise, moderate turbulence was also added to aircraft responses, using the Dryden turbulence model specified in MIL-STD-1797 ${ }^{16}$.

Figure 9 shows simulated aircraft responses for an optimized multisine perturbation input applied to the elevator control surface. The input design is the same one specified for the elevator in Table 1 and Figure 1, and shown in Figure 2. Figure 10 shows the results from 200 Monte Carlo runs, each with different noise and turbulence realizations. Conventional equation-error parameter estimation in the frequency domain was applied, and the modeling was repeated using the explanatory variable sifting process. Figure 10 compares the norm of the vector of true errors for all of the model parameters in the linear dynamic system of Eq. (25) using conventional analysis and the sifting procedure. For the data shown in Figure 10, the improvement in the estimated model parameter accuracy
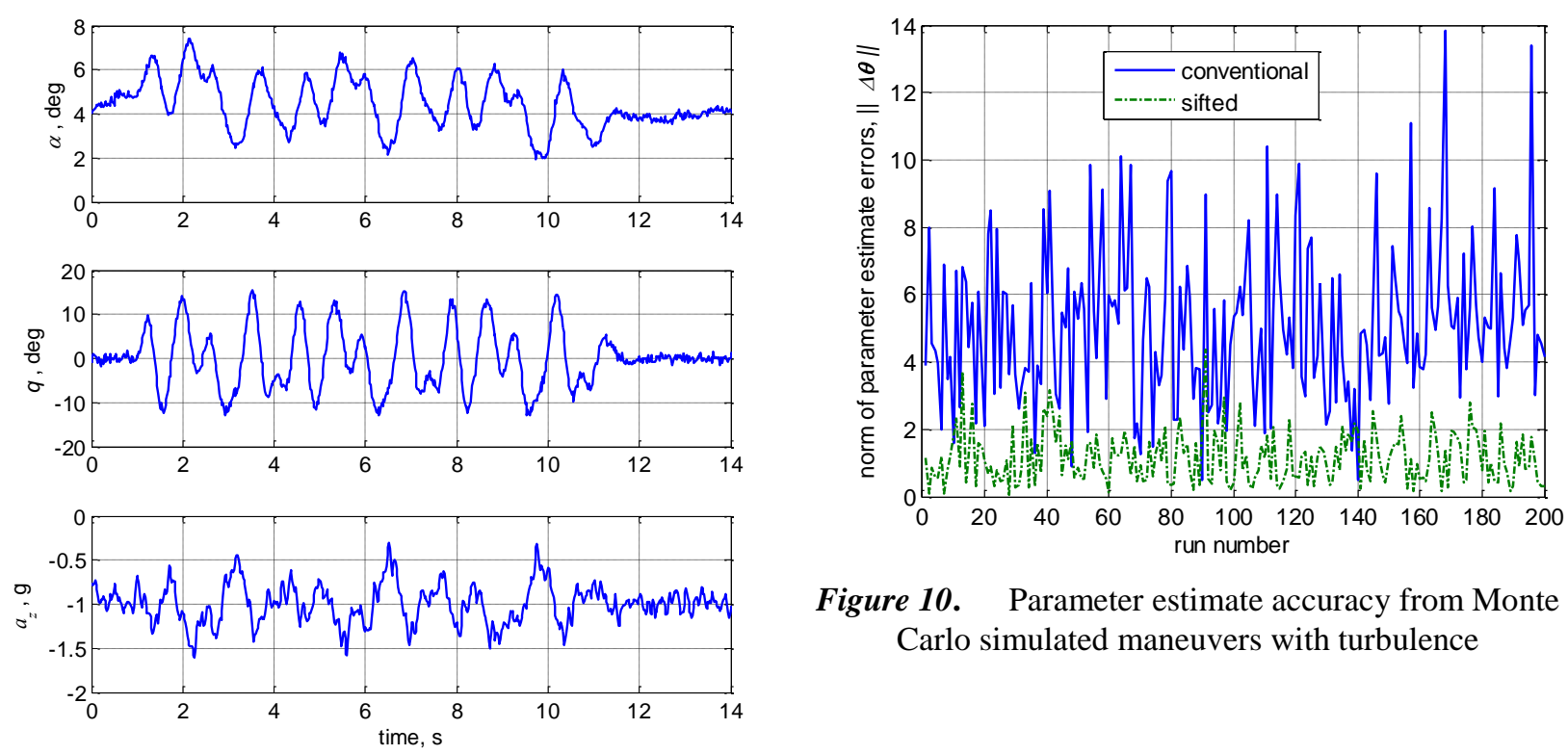

Figure 10. Parameter estimate accuracy from Monte Carlo simulated maneuvers with turbulence

Figure 9. Simulated stability and control flight test data in turbulence

was roughly a factor of 5 and the scatter was reduced by a factor of 3. This Monte Carlo analysis was run many times with similar results.

The Monte Carlo analysis demonstrated that improved frequency-domain data resulting from the sifting process translated directly into more accurate model parameter estimates with less scatter in the estimates. This is consistent with previous work which showed that more accurate model parameter estimates are obtained when the explanatory variables in an equation-error formulation have low noise levels ${ }^{1,15}$.

Further investigations showed that the estimated model parameter accuracy could be further improved by using longer time lengths, which has the effect of further marginalizing transient effects and making the sifting process more accurate. In this case, the model parameter accuracy improved by about 10 percent for each additional $10 \mathrm{sec}$ of excitation. 


\section{Flight Test Results}

Flight test maneuvers similar to the one used for the simulation demonstration were flown on the T-2 subscale jet transport aircraft to collect data for dynamic modeling in both smooth air and turbulent air. Figure 11 shows measured control surface deflections and aircraft responses for one of these maneuvers, where orthogonal optimized multisine perturbation inputs were applied to the elevator, aileron, and rudder control surfaces simultaneously at a nominal flight condition of approximately $4.5 \mathrm{deg}$ angle of attack and $1400 \mathrm{ft}$ altitude in light turbulence.

\section{T-2 FLT 33 C18WT02e}
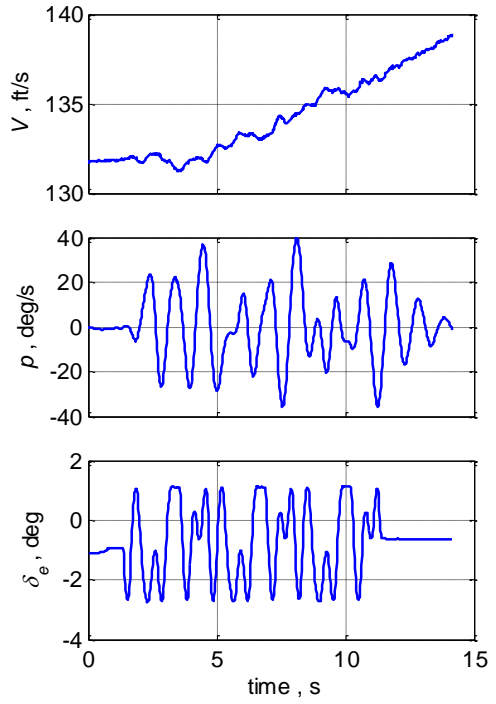
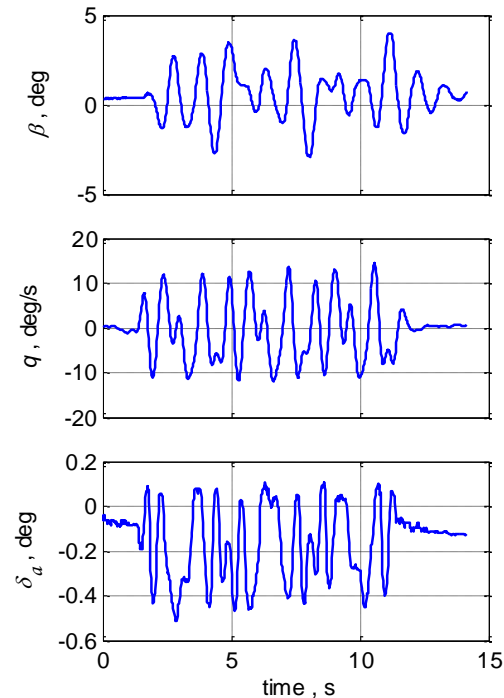
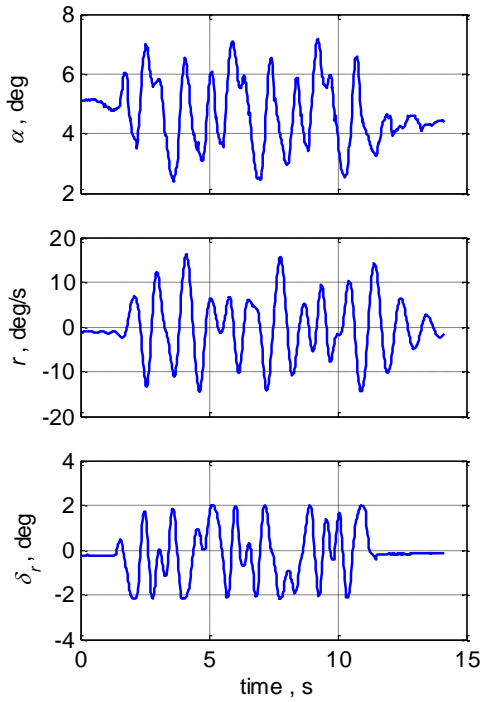

Figure 11. T-2 flight data for a stability and control maneuver in light turbulence

Figure 12 shows parameter estimation results for nondimensional pitching moment model, cf. Eq. (8c). The left column of Figure 12 shows results in light turbulence, the middle column shows results in moderate turbulence, and the right column shows results in severe turbulence, where the turbulence levels where characterized from flight data using the turbulence metric described in Section IV. Parameter estimates for individual repeated maneuvers are shown by different shaped markers, with the estimated 95 percent confidence intervals ( \pm 2 standard error bounds) indicated by attached vertical bars. Each row of plots in Figure 12 shows results for a particular stability or control parameter in the linear model for aerodynamic pitching moment.

The individual groups in each plot of Figure 12 show results using conventional equation-error in the frequency domain, and results using the same technique with sifted frequency-domain data. In all cases, the sifting technique produced slightly less scatter in the parameter estimates, with mean values that were consistent with the results from conventional equation-error in the frequency domain. Note that the parameter estimation results using conventional equation-error in the frequency domain are already quite good, even in severe turbulence. This is mostly due to the automatic filtering in the frequency domain that is inherent in using a limited frequency band for the finite Fourier transform, making the technique robust to turbulence even without applying the sifting technique. The small differences in the mean parameter estimates for maneuvers from flight 15 (severe turbulence) were the result of the maneuvers being executed at a slightly different flight condition compared to the maneuvers from flight 33 (light turbulence) and flight 30 (moderate turbulence). As shown in Figure 11, the excitation time for these maneuvers was only 10 seconds (due to flight test operational constraints), so that the transient response was likely a significant portion of the total response, reducing the effectiveness of the sifting technique. Results for the other stability and control parameter estimates were similar with respect to the consistency of the estimates and the effectiveness of the sifting technique. Tables 3 and 4 provide a full set of mean parameter estimates and standard error uncertainties using the sifting technique applied to T-2 flight data from repeated maneuvers on flight 30 (moderate turbulence). 
T-2 FLT 33 C18WT02

Light Turbulence
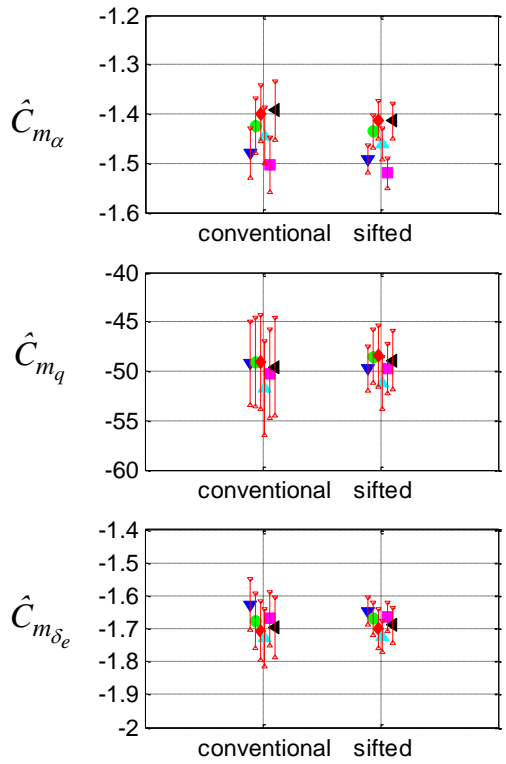

T-2 FLT 30 C18WT02

Moderate Turbulence
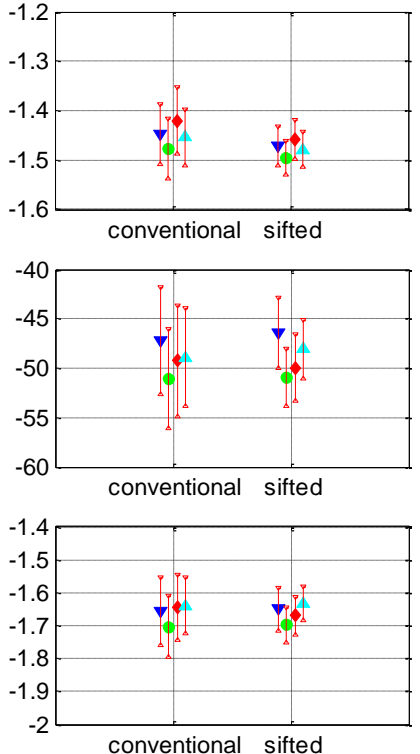

T-2 FLT 15 C21WT02

Severe Turbulence
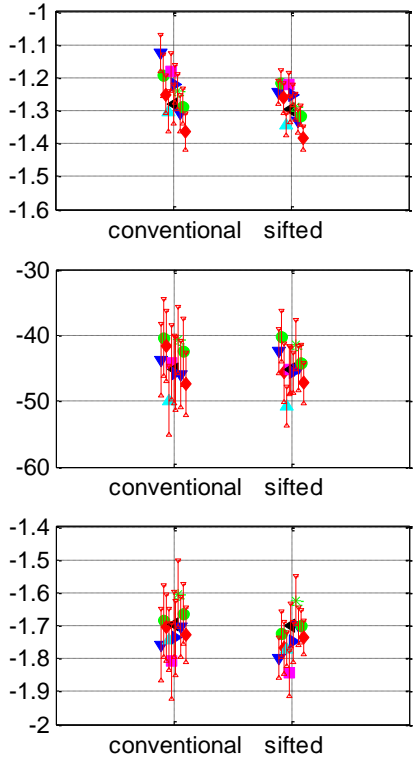

Figure 12. Modeling results from T-2 flight data repeated maneuvers in light, moderate, and severe turbulence

For some of the T-2 flights, amplitudes of orthogonal optimized multisine input excitations were modified based on real-time estimation of the turbulence level. An empirical formula was used to scale the input amplitudes between a set minimum (1) and maximum (2) value, depending linearly on the estimated turbulence level metric estimated in real time. The flight operational procedures necessary to achieve this were successful, as shown by the

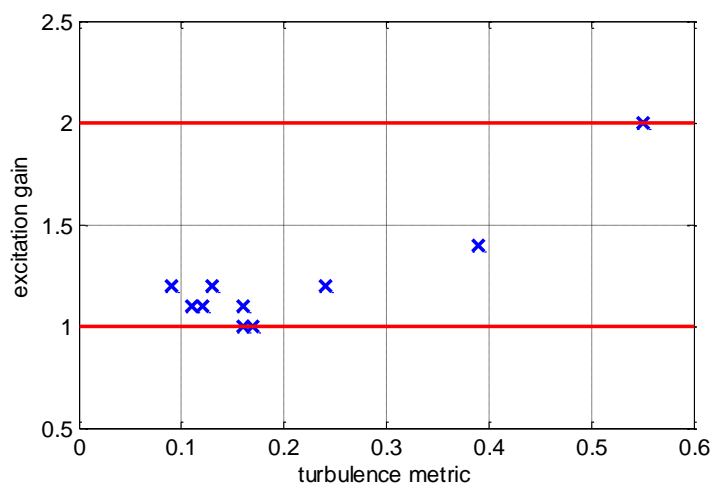

Figure 13. T-2 multisine excitation gain adjustment as a function of estimated turbulence level flight results in Figure 13. The relationship shown in Figure 13 is not exactly linear because a real-time estimate of the turbulence taken near the beginning of each flight was used to set the excitation gain, but the turbulence metric estimated for each entire flight was used to produce Figure 13. The excitation inputs for elevator, aileron, and rudder had nominal amplitudes which were then scaled by the same factor, shown in Figure 13. Estimated parameter accuracy was maintained at increased turbulence levels by increasing excitation input amplitudes, simply because more amplitude for the excitations made the turbulence less relevant in the frequency-domain least squares problem by virtue of increased signal-to-noise ratios at the excitation frequencies and the consequent marginalization of the turbulence. 


\section{Concluding Remarks}

Simulation and flight data from a subscale jet transport aircraft were used to demonstrate a novel approach for accurately identifying aircraft dynamic models in turbulence. Flight results showed that dynamic modeling in turbulence can be done simply and with decent accuracy using conventional equation-error in the frequency domain. However, modeling results in turbulence can be improved by increasing the excitation amplitudes to dominate the turbulence, or by sifting the frequency-domain data to marginalize the effects of turbulence. The resulting dynamic models exhibited good agreement with modeling results obtained at similar flight conditions in smooth air using conventional dynamic modeling methods.

Two fundamental approaches were discussed for dynamic modeling in turbulence: 1) increasing excitation amplitudes to overwhelm increasing turbulence levels, and 2) sifting explanatory variable and output data in the frequency domain. The former could be classified as an experimental method, and the latter as an analytical method. Neither method requires any additional flight hardware or sensors. The experimental approach could be implemented by a pilot, but the analytical approach requires an automated excitation system and relies on using orthogonal optimized multisine excitation inputs. Both approaches are much simpler than the conventional postflight batch filter-error method, and both can be implemented in real time. Future work could include a scheme to combine the experimental and analytical approaches with the turbulence characterization metric developed in this work to optimize real-time dynamic modeling results in any turbulence condition. This could help to make sure that only the minimum required excitation is applied at any time, which has the advantages of improving linearity, minimizing deviations from the planned flight trajectory, and minimizing discomfort for flight crew and passengers. Further flight testing is needed to verify that longer excitation times would produce more accurate parameter estimation results in turbulence using the sifting technique. Developing an analytical method analogous to the sifting technique to account for transient response would improve parameter estimation results in turbulence, and allow the use of shorter excitation times.

Accurate characterization of turbulence levels and associated strategies for overcoming the adverse effects of turbulence could be very useful for improving the efficiency of stability and control flight testing, by improving data quality, reducing the number of maneuver repeats needed for a given level of modeling accuracy, and expanding flight testing opportunities by relaxing turbulence restrictions. This capability also has important implications for aircraft safety, because the flight conditions in which accurate stability and control characterization can be done would be expanded to include turbulent flight conditions. Some environmental hazards such as storms and icing conditions are known to be associated with turbulence. The approaches developed here are simple, can be applied in real time, and have very low computer memory and computational requirements. Applications for this technology include all-weather real-time flight envelope estimation, fault and damage detection, adaptive control, accident investigation, and the capability to monitor changes in the aircraft due to aging, failures, damage, airframe icing, or small differences in manufacturing among aircraft of the same make and model.

\section{Acknowledgments}

The efforts of the AirSTAR flight test team at NASA Langley in building and testing the T-2 aircraft and associated systems, carefully calibrating the instrumentation, and carrying out the flight operations to collect the high-quality flight data used in this study, are gratefully acknowledged. Research in Aircraft System Identification is funded by the NASA Aviation Safety Program, Vehicle Systems Safety Technologies (VSST) project.

\section{References}

\footnotetext{
${ }^{1}$ Klein, V. and Morelli, E.A., Aircraft System Identification - Theory and Practice, AIAA Education Series, AIAA, Reston, VA, 2006.

${ }^{2}$ Maine, R.E. and Iliff, K.W. "Formulation and Implementation of a Practical Algorithm for Parameter Estimation with Process and Measurement Noise," SIAM Journal of Applied Mathematics, Vol. 41, No. 3, 1981, pp. 558-579.

${ }^{3}$ Jategaonkar R.V. and Plaetschke, E. "Algorithms for aircraft parameter estimation accounting for process and measurement noise", Journal of Aircraft, Vol. 26, No. 4, 1989, pp. 360-372.

${ }^{4}$ Martos, B. and Morelli, E.A. "Using Indirect Turbulence Measurements for Real-Time Parameter Estimation in Turbulent Air,", AIAA Atmospheric Flight Mechanics Conference, Minneapolis, MN, August 2012.

${ }^{5}$ Shah, G.H., Cunningham, K., Foster, J.V., Fremaux, C.M., Stewart, E.C., Wilborn, J.E., Gato, W., Pratt, D.W., "WindTunnel Investigation of Commercial Transport Aircraft Aerodynamics at Extreme Flight Conditions", SAE 2002-01-2912, World Aviation Congress \& Display, Phoenix, AZ, November 2002.
} 
${ }^{6}$ Murch, A.M. and Foster, J.V. "Recent NASA Research on Aerodynamic Modeling of Post-Stall and Spin Dynamics of Large Transport Airplanes", AIAA-2007-463-253, 45 ${ }^{\text {th }}$ AIAA Aerospace Sciences Meeting and Exhibit, Reno, NV, January 2007.

${ }^{7}$ Morelli, E.A. "Multiple Input Design for Real-Time Parameter Estimation in the Frequency Domain," Paper REG-360, 13 IFAC Symposium on System Identification, Rotterdam, The Netherlands, August 2003.

${ }^{8}$ Morelli, E.A. "Flight-Test Experiment Design for Characterizing Stability and Control of Hypersonic Vehicles," Journal of Guidance, Control, and Dynamics, Vol. 32, No. 3, May-June 2009, pp. 949-959.

${ }^{9}$ Morelli, E.A. and Smith, M.S. "Real-Time Dynamic Modeling - Data Information Requirements and Flight Test Results," Journal of Aircraft, Vol. 46, No. 6, November-December 2009, pp. 1894-1905.

${ }^{10}$ Morelli, E.A. "Flight Test Maneuver Design for Efficient Aerodynamic Modeling," AIAA 2011-6672, AIAA Atmospheric Flight Mechanics Conference, Portland, OR, August 2011.

${ }^{11}$ Press, W.H., S.A. Teukolsky, W.T. Vettering, and B.R. Flannery Numerical Recipes in FORTRAN: The Art of Scientific Computing, $2^{\text {nd }}$ Ed., Cambridge University Press, New York, NY, 1992, Chapter 10.

${ }^{12}$ Jordan, Thomas L., Langford, William M., Hill, Jeffrey S.; “Airborne Subscale Transport Aircraft Research Testbed: Aircraft Model Development”, AIAA 2005-6432, AIAA Guidance, Navigation, and Control Conference and Exhibit, San Francisco, CA, August 2005.

${ }^{13}$ Jordan, Thomas L., Foster, John V., Bailey, Roger M., and Belcastro, Christine M., "AirSTAR: A UAV Platform for Flight Dynamics and Control System Testing," AIAA-2006-3307, 25th AIAA Aerodynamic Measurement Technology and Ground Testing Conference, San Francisco, CA, June 2006.

${ }^{14}$ Murch, A. M., "A Flight Control System Architecture for the NASA AirSTAR Flight Test Facility," AIAA 2008-6990, AIAA Guidance, Navigation, and Control Conference and Exhibit, Honolulu, HI, 2008.

${ }^{15}$ Morelli, E.A., "Practical Aspects of the Equation-Error Method for Aircraft Parameter Estimation," AIAA-2006-6144, AIAA Atmospheric Flight Mechanics Conference, Keystone, CO, August 2006.

${ }^{16}$ Military Standard - Flying Qualities of Piloted Aircraft, MIL-STD-1797A, January 1990.

Table 1 Multiple input design for the T-2 subscale jet transport aircraft, level flight, $V_{o}=80 \mathrm{kts}, \alpha_{o}=5 \mathrm{deg}, T=10 \mathrm{sec}$

\begin{tabular}{|c|c|c|c|c|c|}
\hline Input & $A(\mathrm{deg})$ & $A_{k}(\operatorname{deg})$ & $k$ & $\phi_{k}(\mathrm{rad})$ & $R P F$ \\
\hline \multirow{7}{*}{$\delta_{e}$} & \multirow{7}{*}{1.0} & 0.3162 & 3 & 2.9478 & \multirow{7}{*}{1.0} \\
\hline & & 0.3873 & 6 & 0.6008 & \\
\hline & & 0.4472 & 9 & -2.6991 & \\
\hline & & 0.4472 & 12 & -1.6517 & \\
\hline & & 0.3873 & 15 & 2.6902 & \\
\hline & & 0.3162 & 18 & 2.0873 & \\
\hline & & 0.3162 & 21 & -2.8619 & \\
\hline \multirow{7}{*}{$\delta_{r}$} & \multirow{7}{*}{1.0} & 0.3162 & 2 & 2.8435 & \multirow{7}{*}{1.14} \\
\hline & & 0.3873 & 5 & 2.5259 & \\
\hline & & 0.4472 & 8 & 2.7562 & \\
\hline & & 0.4472 & 11 & -0.5132 & \\
\hline & & 0.3873 & 14 & -0.7433 & \\
\hline & & 0.3162 & 17 & 2.3959 & \\
\hline & & 0.3162 & 20 & -0.7581 & \\
\hline \multirow{7}{*}{$\delta_{a}$} & \multirow{7}{*}{1.0} & 0.3780 & 4 & 1.5438 & \multirow{7}{*}{1.15} \\
\hline & & 0.3780 & 7 & -1.6413 & \\
\hline & & 0.3780 & 10 & 1.2011 & \\
\hline & & 0.3780 & 13 & 1.0767 & \\
\hline & & 0.3780 & 16 & -2.3373 & \\
\hline & & 0.3780 & 19 & -2.3327 & \\
\hline & & 0.3780 & 22 & -2.7602 & \\
\hline
\end{tabular}


Table 2. T-2 aircraft geometry and nominal mass properties

\begin{tabular}{|c|l|}
\hline $\bar{c}, \mathrm{ft}$ & 0.915 \\
\hline$b, \mathrm{ft}$ & 6.849 \\
\hline$S, \mathrm{ft}^{2}$ & 5.902 \\
\hline$x_{o}$, in & 57.30 \\
\hline$y_{o}$, in & 0.000 \\
\hline$z_{o}$, in & 11.28 \\
\hline$x_{c g}$, in & 56.63 \\
\hline$y_{c g}$, in & 0.000 \\
\hline$z_{c g}$, in & 11.43 \\
\hline$m$, slugs & 1.585 \\
\hline$I_{x}$, slugs-ft & 1.179 \\
\hline$I_{y}$, slugs- $\mathrm{ft}^{2}$ & 4.520 \\
\hline$I_{z}$, slugs- $\mathrm{ft}^{2}$ & 5.527 \\
\hline$I_{x z}$, slugs- $\mathrm{ft}^{2}$ & 0.211 \\
\hline \hline
\end{tabular}

Table 3. Parameter estimates and uncertainties for longitudinal model identified from T-2 flight data

\begin{tabular}{|c|c|c|c|c|c|}
\hline \hline $\begin{array}{c}C_{X} \text { Model } \\
\text { Parameter }\end{array}$ & $\begin{array}{c}\text { Estimate } \\
\pm \text { Std. Error }\end{array}$ & $\begin{array}{c}C_{Z} \text { Model } \\
\text { Parameter }\end{array}$ & $\begin{array}{c}\text { Estimate } \\
\pm \text { Std. Error }\end{array}$ & $\begin{array}{c}C_{m} \text { Model } \\
\text { Parameter }\end{array}$ & $\begin{array}{c}\text { Estimate } \\
\pm \text { Std. Error }\end{array}$ \\
\hline$C_{X_{\alpha}}$ & $0.567 \pm 0.009$ & $C_{Z_{\alpha}}$ & $-4.021 \pm 0.040$ & $C_{m_{\alpha}}$ & $-1.476 \pm 0.015$ \\
\hline$C_{X_{\delta_{e}}}$ & $-0.066 \pm 0.006$ & $C_{Z_{\delta_{e}}}$ & $0.222 \pm 0.028$ & $C_{m_{q}}$ & $-48.85 \pm 1.96$ \\
\hline & & & $C_{m_{\delta_{e}}}$ & $-1.663 \pm 0.027$ \\
\hline \hline
\end{tabular}

Table 4. Parameter estimates and uncertainties for lateral model identified from T-2 flight data

\begin{tabular}{|c|c|c|c|c|c|}
\hline \hline $\begin{array}{c}C_{Y} \text { Model } \\
\text { Parameter }\end{array}$ & $\begin{array}{c}\text { Estimate } \\
\pm \text { Std. Error }\end{array}$ & $\begin{array}{c}C_{l} \text { Model } \\
\text { Parameter }\end{array}$ & $\begin{array}{c}\text { Estimate } \\
\pm \text { Std. Error }\end{array}$ & $\begin{array}{c}C_{n} \text { Model } \\
\text { Parameter }\end{array}$ & $\begin{array}{c}\text { Estimate } \\
\pm \text { Std. Error }\end{array}$ \\
\hline$C_{Y_{\beta}}$ & $-0.770 \pm 0.010$ & $C_{l_{\beta}}$ & $-0.112 \pm 0.025$ & $C_{n_{\beta}}$ & $0.219 \pm 0.003$ \\
\hline$C_{Y_{r}}$ & $0.618 \pm 0.155$ & $C_{l_{p}}$ & $-0.234 \pm 0.099$ & $C_{n_{r}}$ & $-0.459 \pm 0.055$ \\
\hline$C_{Y_{\delta_{r}}}$ & $0.153 \pm 0.013$ & $C_{l_{r}}$ & $-0.461 \pm 0.132$ & $C_{n_{\delta_{r}}}$ & $-0.160 \pm 0.005$ \\
\hline & & $C_{l_{\delta_{a}}}$ & $-0.047 \pm 0.019$ & & \\
\hline & & $C_{l_{\delta_{r}}}$ & $0.042 \pm 0.004$ & & \\
\hline \hline
\end{tabular}

